(2) Open Access Full Text Article

METHODOLOGY

\title{
Spontaneous peritonitis in critically ill cirrhotic patients: a diagnostic algorithm for clinicians and future perspectives
}

This article was published in the following Dove Press journal:

Therapeutics and Clinical Risk Management

16 October 2017

Number of times this article has been viewed

\author{
Marco Fiore' \\ Alberto Enrico Maraolo ${ }^{2}$ \\ Sebastiano Leone ${ }^{3}$ \\ Ivan Gentile ${ }^{2}$ \\ Arturo Cuomo 4 \\ Vincenzo Schiavone ${ }^{5}$ \\ Sabrina Bimonte ${ }^{4}$ \\ Maria Caterina Pace' \\ Marco Cascella ${ }^{4}$ \\ 'Department of Anaesthesiological, \\ Surgical and Emergency Sciences, \\ University of Campania "Luigi \\ Vanvitelli", Naples, Italy; 'Department \\ of Clinical Medicine and Surgery, \\ Section of Infectious Diseases, \\ University of Naples Federico II, \\ Naples, Italy; ${ }^{3}$ Division of Infectious \\ Diseases, "San Giuseppe Moscati" \\ Hospital, Avellino, Italy; ${ }^{4}$ Division of \\ Anesthesia, Department of Anesthesia \\ and Pain Medicine, Istituto Nazionale \\ Tumori - IRCCS - Fondazione \\ G. Pascale, Naples, Italy; ${ }^{5}$ Division \\ of Anesthesia and Intensive Care, \\ Hospital "Pineta Grande", Castel \\ Volturno, Italy
}

Correspondence: Marco Cascella Division of Anesthesia, Department of Anesthesia and Pain Medicine, Istituto Nazionale Tumori - IRCCS - Fondazione G. Pascale, Via Mariano Semmola, Naples 80I3I, Italy Email m.cascella@istitutotumori.na.it
Abstract: Spontaneous peritonitis (SP) is the most common infection among decompensated end-stage liver disease patients. SP is the infection of ascitic fluid (neutrophil ascitic count $\geq 250 / \mathrm{mL}$ ) without an alternative focus of abdominal infection. According to the causative agent, clinicians can make the diagnosis of spontaneous bacterial peritonitis or spontaneous fungal peritonitis. The mortality rate is very high, ranging from one-fifth of the patients with spontaneous bacterial peritonitis to four-fifths of the patients with spontaneous fungal peritonitis. An immediate and accurate diagnosis can improve the outcome in end-stage liver disease patients. The aim of this work is to provide physicians with a practical diagnostic guidance for SP diagnosis according to current evidence, in order to improve the management of cirrhotic patients with infected ascitic fluid.

Keywords: cirrhosis, spontaneous bacterial peritonitis, spontaneous fungal peritonitis, bacteriascites, fungal ascites

\section{Introduction}

Advanced liver disease is a concern in developed countries, representing the 14th most frequent cause of death globally and even the fourth in regions such central Europe. ${ }^{1}$ One of the most important reasons of hepatic decompensation in cirrhotic patients is bacterial infections, which currently are deemed as a distinct prognostic stage of liver disease, worsening the outcome regardless of illness severity. ${ }^{2}$ Unfortunately, in cirrhotic patients the diagnosis of bacterial infections is often very difficult. At any rate, they are the main inciting factor of the so-called acute-on-chronic liver failure, a clinical entity associated with organ failures and notable short-term mortality. ${ }^{3}$

Spontaneous peritonitis (SP) is an infection of ascites in cirrhotic patients without an alternative intra-abdominal focus of infection; when a cultural examination is performed on ascites, the growth of bacteria leads to a diagnosis of spontaneous bacterial peritonitis (SBP) and the growth of fungi leads to a diagnosis of spontaneous fungal peritonitis (SFP). ${ }^{4} \mathrm{SP}$ is the most common infection responsible for sepsisinduced acute-on-chronic liver failure. ${ }^{5}$ Moreover, SP and urinary tract infections are the most common infections among decompensated end-stage liver disease (ESLD) patients. ${ }^{6}$ Bacterial infections are a common complication in patients with ESLD, and they occur either upon hospital admission or during the course of hospitalization. Up to three-fifths of hospitalized ESLD patients present with an infection. SBP is the most frequent bacterial infection in ESLD patients, accounting for one-third of all reported bacterial infections in hospitalized ESLD patients. ${ }^{7}$ SP diagnosis relies on 
ascitic fluid cell analysis (polymorphonuclear [PMN] cell count $\geq 250 / \mathrm{mm}^{3}$ ). Microbiological culture (either from ascitic fluid or, as recently proposed, from bloodstream) enables identification of the etiological agent. ${ }^{8}$

Conventional diagnostic methods based on the use of culture remain the mainstay of diagnosis of SP. According to the identification of bacteria or fungi, physicians can make the diagnosis of SBP or SFP. ${ }^{9}$ However, the positivity of ascitic fluid culture is not constant, generally lower than $50 \%,{ }^{10}$ and blood culture is not performed in most cases; thereby, many cases are classified as culture-negative SP.

The presence of microbes in the ascites fluid and/or blood is the result of the fundamental pathogenic mechanism underlying SP consisting in the microbial translocation, from the gut to systemic circulation through the mesenteric nodes. ${ }^{11}$ SP mortality is high, ranging from more than one-fifth of ESLD patients for $\mathrm{SBP}^{10}$ to approximately four fifth of ESLD patients for SFP. ${ }^{9}$

Unfortunately, SBP mortality has not diminished in recent years, probably due to the changing microbiological profile. $^{12}$

Timely diagnosis is crucial for the survival of critically ill cirrhotic patients because, as recently stated by the Cooperative Antimicrobial Therapy of Septic Shock Group, each hour of delay in appropriate treatment is associated with a two-fold increase in hospital mortality. ${ }^{8}$

The purpose of this work is to give clinicians an easy diagnostic guidance in the SP diagnosis according to current evidence in order to improve the survival of cirrhotic patients with infected ascitic fluid.

\section{Current evidence}

The diagnosis of SP is based on a PMN leukocyte count (PMN $\left.\geq 250 / \mathrm{mm}^{3}\right) .{ }^{6}$ However, manual microscopic PMN counting is a time-consuming procedure, even demanding the necessary expertise in order to reduce both the intraand interobserver variability. ${ }^{13}$ As a result, manual count sometimes is not carried out in general hospitals, particularly outside office hours. ${ }^{13}$ This can imply an unacceptable diagnostic and therapeutic delay, doubling the mortality rate of involved patients. ${ }^{8}$

Other strategies have been developed to perform a simple and rapid bedside SP diagnosis, especially for ESLD patients treated in an ambulatory setting. The most promising is the Periscreen strip, which is a test assessing the leukocyte esterase activity based on four different chemical pads which react (color change) when immersed in an ascitic fluid sample. The colorimetric gradation varies from white, light yellow, to purple as "negative", "trace", "small", or "large". Periscreen strip for SP using the "trace" (ie, white vs colored) threshold is remarkable (sensitivity $100 \%$ and negative-predictive value $100 \%$ ) in outpatients, but also in inpatients (sensitivity $89.5 \%$ and negative-predictive value 97.9\%). ${ }^{13}$ The Periscreen strip has been validated in a large population of decompensated ESLD patients. ${ }^{13}$ In addition, it should be performed in "symptomatic" ESLD outpatients when at least one or more of the following criteria compatible with peritonitis is present: abdominal tenderness, fever $\left(>38^{\circ} \mathrm{C}\right)$ or low body temperature $\left(<36.5^{\circ} \mathrm{C}\right)$, shiver, new onset or worsening of a preexisting encephalopathy, recent history of gastrointestinal bleeding, and/or hypotension. ${ }^{14}$ Therefore, if the Periscreen strip is positive, indicating significant leukocyte esterase activity, the patient requires early empiric antibiotic therapy (EAT), with the comparison of this result with cytology, and immediate microbiological culture.

Irrespective of the first diagnostic approach (manual microscopic PMN counting versus Periscreen strip), microbiological examination through the culture of ascitic fluid and blood is a fundamental step, ${ }^{8,15}$ although the results are not constantly positive. ${ }^{16}$ It should be systematically carried out at the bedside by inoculation of $10 \mathrm{~mL}$ of blood as well as ascitic fluid in both aerobic and anaerobic blood culture vials. ${ }^{15}$ Obtaining ascitic fluid for diagnostic/therapeutic purposes in ESLD patients hospitalized for evaluation and management of symptoms related to ascites is associated with increased short-term survival, although in the United States ESLD patients hospitalized for ascites or encephalopathy often do not receive a paracentesis. ${ }^{17}$ On the other hand, delayed paracentesis is associated with increased mortality in SBP. As shown by Kim et al, ${ }^{18}$ every hour of delay in paracentesis is associated with $3 \%$ increase in mortality. Moreover, paracentesis must be repeated after 48 hours of treatment in patients who do not show significant improvement, ${ }^{15,19}$ and treatment failure is believed to occur if the absolute PMN count has decreased by $<25 \%$ on 48 -hour repeat paracentesis. Therapy failure is to be suspected if PMN count in ascitic fluid does not show a decrease of at least $25 \%$ compared with the pretreatment value after 48 hours of antimicrobial treatment. ${ }^{15}$

Follow-up paracentesis should not be mandatory if the patient properly responds to the antibiotic therapy. After 48 hours treatment without any effects, the clinician is able to suspect an SFP since hospitalization is linked to an increased risk of contracting SFP. ${ }^{9,20}$ Therefore, a new culture (for bacteria and fungi) of ascitic fluid and blood should be performed. 


\section{The algorithm}

The algorithm we propose (Figure 1) is exclusively for adults in whom cirrhosis is documented and the clinical suspicion of SP is high. The aim of this algorithm is to standardize antimicrobial or non-antimicrobial management of SP, and not to provide a complete data-supported approach to manage ascites. This approach may be helpful in order to anticipate SP diagnosis, help propose an appropriate antimicrobials therapy, and, finally, lead to a reduction of mortality and antimicrobial resistance. The first step in the suspicion of SP consists of the execution of the Periscreen strip test. This strategy allows limiting the use of antibiotics and starting a timely EAT. Microbiology culture from blood and ascitic fluid are, according to the current evidence, the gold standard to diagnose bacterial/fungal infections, but the results are sometimes available only after more than 48 hours, often resulting in either a therapeutic delay or overtreatment with a broad-spectrum empirical regimen. ${ }^{21}$ The high negativepredictive value of the Periscreen strip test permits to rule out SP in the short-term. ${ }^{13}$ In the case of positivity, further examinations are, at any rate, mandatory, especially the PMN leukocyte count of ascitic fluid and microbiological cultures of ascitic fluid and blood, in order to try to identify the causative agent of the infection. Up to $45 \%$ of patients with SBP have infections by bacteria resistant to first-line antibiotics. ${ }^{22}$ Furthermore, fungal infections in cirrhotic patients (SFP in this particular case) are a matter of concern. ${ }^{23}$ Recently, a meta-analysis showed that the risk of SFP in nosocomial SP was $5 \%$ (95\% confidence interval $1 \%-10 \%$ ) with a significant heterogeneity among studies $(p<0.001$, $\mathrm{I}=78 \%)$; the risk of SFP in non-nosocomial SP was $1 \%(95 \%$ confidence interval $0.1 \%-3 \%) .{ }^{20}$ In a German study, SFP was seen in about two-fifths of critically ill patients with ESLD and SP, either nosocomial or not-nosocomial, with a positive ascitic fluid culture. ${ }^{24}$

The choice of the EAT is crucial, and equally important is the decision regarding the escalation or the de-escalation of the initial regimen prescribed, making the development of new, better, and faster diagnostic techniques of utmost importance. ${ }^{21}$ It is important to establish the setting wherein the infection occurs: nosocomial or health care-related infections are more likely to be induced by multidrug-resistant organisms than community-acquired infections. ${ }^{25}$ Nevertheless, the local epidemiology is always to be taken into account, so as to avoid empiric use of broad-spectrum agents in contexts with a very low prevalence of resistant pathogens. ${ }^{26}$

\section{Areas of uncertainty}

This paper focuses on the diagnosis of SP (based on a PMN $\geq 250 / \mathrm{mm}^{3}$ ) and does not take into account bacteriascites, which is a variant of SP where a single bacterial organism grows in ascitic fluid but the number of PMN is $<250 / \mathrm{mm}^{3}$. While three decades ago bacterascites was considered a pathologically benign condition not requiring antimicrobial treatment, ${ }^{27}$ currently it seems to be associated with a high mortality rate. Indeed, Piroth et $\mathrm{al}^{28}$ observed, in patients with bacterascites, a mortality rate close to one-fifth of patients.

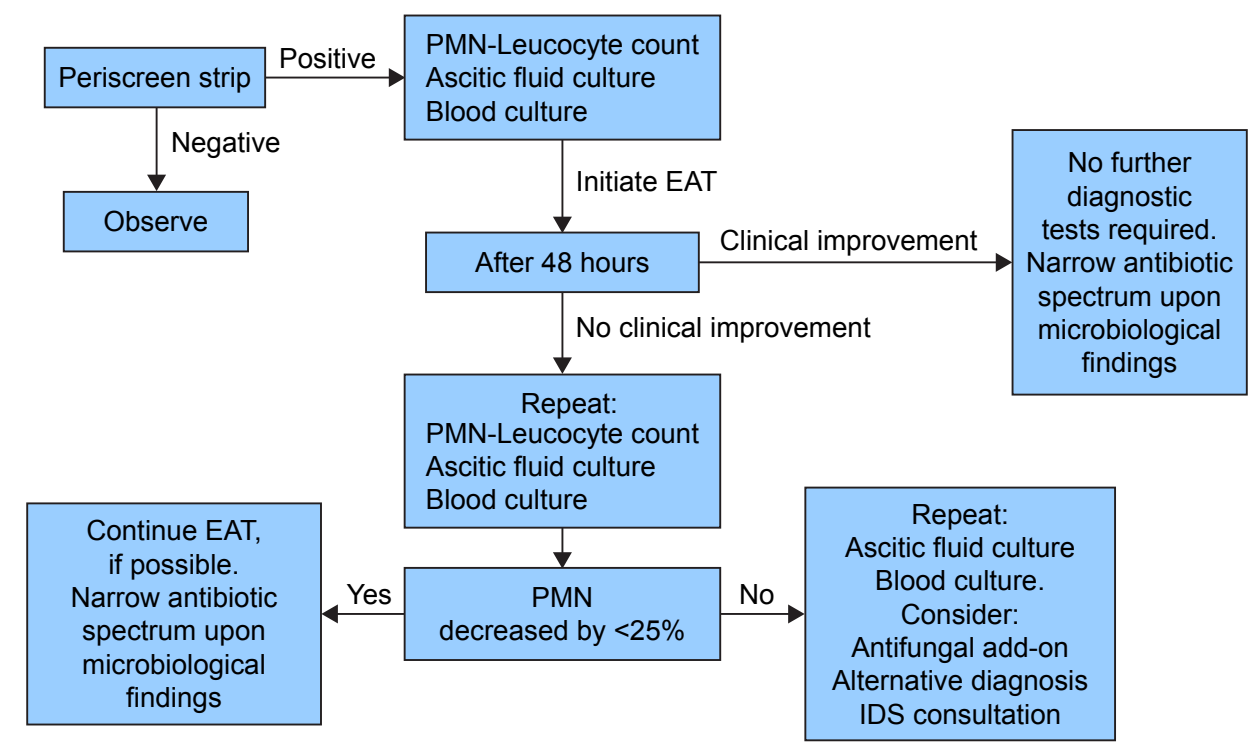

Figure I The algorithm.

Abbreviations: EAT, empiric antibiotic therapy; IDS, International Diagnostic Solutions; PMN, polymorphonuclear. 
Fungal ascites (a fungus growing in ascitic fluid but with total patient PMN PMN $<250 / \mathrm{mm}^{3}$ ) has a higher mortality rate than bacteriascites. ${ }^{9}$ The mortality rate of fungal ascites is close to $50 \%$ of infected patients within 1 year after hospital discharge..$^{29}$ These considerations raise questions about the current definition of SP.

Consequently, future studies are needed to understand if bacteriascites/fungal ascites antimicrobial treatment improves survival in this clinical setting.

\section{Future perspectives}

Host response-based serum biomarkers such as procalcitonin (PCT) and c-reactive protein are the most frequently used serum markers for the early detection (as well as to test the severity) of bacterial infection, to complement direct pathogen detection. ${ }^{30}$ Unfortunately, their sensitivity and specificity in cirrhotic patients is impaired by a wide array of factors, among them the stimulus represented by the bacterial translocation through the gut, which, irrespective of real infection, stands out. ${ }^{6}$

Recently, new markers have been proposed for the early diagnosis of peritonitis, including lactoferrin, expression of CD64 on neutrophils (CD64 index), serum PCT, and ascitic calprotectin. ${ }^{30-35}$

Lactoferrin may be useful to diagnose SBP in ESLD patients; the limit of this method is that elevated ascitic fluid lactoferrin level may also be related to hepatocellular carcinoma in ESLD patients without SBP. ${ }^{31}$

Another potential marker of SBP in cirrhotic patients is the CD64 index, and this could be used as a more effective marker of PMN counts to modulate antimicrobial therapy. ${ }^{32}$ The aforementioned PCT is a valid test to diagnose SBP, but, as shown by a recent meta-analysis, it cannot serve as a standalone examination and needs further clinical or laboratory findings. ${ }^{33}$ Ascitic calprotectin is an accurate marker for $\mathrm{SP},{ }^{34}$ especially when it is combined with serum procalcitonin, ${ }^{35}$ and the combined use of these two markers is very promising.

Notwithstanding, these tools present an important limitation: they do not permit the etiological diagnosis of SP. On the other hand, methods able to detect a few bacteria per milliliter might potentially serve as a game-changer in the microbiological field: for instance, label-free bimodal waveguide immunosensor demonstrates this property, and in the future could possibly become a very user-friendly tool for clinical microbiologists. ${ }^{36}$

Meanwhile, the objective of rapid diagnostic platforms is to provide a (near) point-of-care system to yield microbiological results within 1-2 hours: potential pitfalls could be the clinical significance of detected bacteria in the context of massive gut bacterial translocation (when no clear signs and/or symptoms of infection are present) and the limited number of the pathogens identified by the panels. ${ }^{37,38}$

\section{Conclusion}

A timely diagnosis is fundamental to guarantee the best management of patients presenting with SP, which is usually caused by bacteria, but, in a not-negligible percentage, also by fungi. Unfortunately, it can take hours to obtain results of PMN cell number from the ascitic fluid; even more time is necessary to have information related to microbiological tests. Therefore, a correct diagnostic algorithm of SP needs to be implemented so as to avoid a potentially fatal delay in starting a course of antibiotic therapy. This algorithm should include a fast and easy diagnostic method such as the Periscreen test. Of course, the Periscreen cannot serve as a standalone test, but it can be the first step of a diagnostic workup for a newly hospitalized patient aimed at identifying the etiologic cause of the infection. The choice of empiric antimicrobials should rely on local epidemiological data; in areas with high rates of multidrug-resistant organisms, broad-spectrum therapy is appropriate, which then has to be converted to a targeted treatment in case of specific bacterial or fungal isolation.

The algorithm per se does not change the choice of the empirical antibiotic treatment; its purpose is to limit the use of antibiotics and encourage narrowing of the antibiotic spectrum as soon as possible in order to decrease antimicrobial expose and lead to substantial cost savings. ${ }^{39}$

\section{Acknowledgments}

The authors are grateful to Dr Alessandra Trocino and Cristina Romano from the National Cancer Institute of Naples for providing excellent bibliographic service and assistance.

\section{Author contributions}

MF and MCP designed the study; MF, MC, and AEM collected the data. IG, AC, SB, VS, and SL contributed to the conception of the study. All authors contributed toward data analysis, drafting and critically revising the paper, gave final approval of the version to be published, and agree to be accountable for all aspects of the work.

\section{Disclosure}

The authors report no conflicts of interest in this work.

\section{References}

1. Tsochatzis EA, Bosch J, Burroughs AK. Liver cirrhosis. Lancet. 2014; 383(9930):1749-1761. 
2. Dionigi E, Garcovich M, Borzio M, et al. Bacterial infections change natural history of cirrhosis irrespective of liver disease severity. Am J Gastroenterol. 2017;12:588-596.

3. Jalan R, Yurdaydin C, Bajaj JS, et al. World gastroenterology organization working party. Toward an improved definition of acute-on-chronic liver failure. Gastroenterology. 2014;147:4-10.

4. Fiore M, Maraolo AE, Gentile I, et al. Nosocomial spontaneous bacterial peritonitis antibiotic treatment in the era of multi-drug resistance pathogens: a systematic review. World J Gastroenterol. 2017;23: 4654-4660.

5. Moreau R, Jalan R, Gines P, et al. Acute-on-chronic liver failure is a distinct syndrome that develops in patients with acute decompensation of cirrhosis. Gastroenterology. 2013;144:1426-1437.

6. Jalan R, Fernandez J, Wiest R, et al. Bacterial infections in cirrhosis: a position statement based on the EASL Special Conference 2013. J Hepatol. 2014;60:1310-1324.

7. Wiest R, Krag A, Gerbes A. Spontaneous bacterial peritonitis: recent guidelines and beyond. Gut. 2012;61(2):297-310.

8. Karvellas CJ, Abraldes JG, Arabi YM, et al. Cooperative Antimicrobial Therapy of Septic Shock (CATSS) Database Research Group. Appropriate and timely antimicrobial therapy in cirrhotic patients with spontaneous bacterial peritonitis-associated septic shock: a retrospective cohort study. Aliment Pharmacol Ther. 2015;41:747-757.

9. Fiore M, Leone S. Spontaneous fungal peritonitis: epidemiology, current evidence and future prospective. World J Gastroenterol. 2016;22 $7742-7747$.

10. European Association for the Study of the Liver. EASL clinical practice guidelines on the management of ascites, spontaneous bacterial peritonitis, and hepatorenal syndrome in cirrhosis. J Hepatol. 2010; 53:397-417.

11. Lachar J, Bajaj JS. Changes in the microbiome in cirrhosis and relationship to complications: hepatic encephalopathy, spontaneous bacterial peritonitis, and sepsis. Semin Liver Dis. 2016;36:327-330.

12. Oliveira AM, Branco JC, Barosa R, et al. Clinical and microbiological characteristics associated with mortality in spontaneous bacterial peritonitis: a multicenter cohort study. Eur J Gastroenterol Hepatol. 2016; 28:1216-1222.

13. Thévenot T, Briot C, Macé V, et al. CFEHTP, ANGH and the PerDRISLA study group. The Periscreen strip is highly efficient for the exclusion of spontaneous bacterial peritonitis in cirrhotic outpatients. Am J Gastroenterol. 2016;111:1402-1409.

14. Nousbaum JB, Cadranel JF, Nahon P, et al. Club Francophone pour 1'Etude de 1'Hypertension Portale; Association Nationale des HépatoGastroentérologues des Hôpitaux Généraux de France. Diagnostic accuracy of the Multistix 8 SG reagent strip in diagnosis of spontaneous bacterial peritonitis. Hepatology. 2007;45:1275-1281.

15. Piano S, Fasolato S, Salinas F, et al. The empirical antibiotic treatment of nosocomial spontaneous bacterial peritonitis: results of a randomized, controlled clinical trial. Hepatology. 2016;63:1299-1309.

16. Pericleous M, Sarnowski A, Moore A, et al. The clinical management of abdominal ascites, spontaneous bacterial peritonitis and hepatorenal syndrome: a review of current guidelines and recommendations. Eur J Gastroenterol Hepatol. 2016;28(3):e10-e18.

17. Orman ES, Hayashi PH, Bataller R, et al. Paracentesis is associated with reduced mortality in patients hospitalized with cirrhosis and ascites. Clin Gastroenterol Hepatol. 2014;12:496-503.

18. Kim JJ, Tsukamoto MM, Mathur AK, et al. Delayed paracentesis is associated with increased in-hospital mortality in patients with spontaneous bacterial peritonitis. Am J Gastroenterol. 2014;109: 1436-1442.

19. Guarner C, Soriano G. Spontaneous bacterial peritonitis. Semin Liver Dis. 1997; 17:203-217.
20. Fiore M, Chiodini P, Pota V, et al. Risk of spontaneous fungal peritonitis (SFP) in hospitalized cirrhotic patients with ascites: a systematic review of observational studies and meta-analysis. Minerva Anestesiol. Epub 2017 Jul 20.

21. Shallcross L, O'Brien A. Antimicrobial resistance in liver disease: better diagnostics are needed. Lancet Gastroenterol Hepatol. 2017;2: 151-153.

22. Fernández J, Bert F, Nicolas-Chanoine MH. The challenges of multidrug-resistance in hepatology. J Hepatol. 2016;65:1043-1054.

23. Alexopoulou A, Vasilieva L, Agiasotelli D, et al. Fungal infections in patients with cirrhosis. J Hepatol. 2015;63:1043-1045.

24. Lahmer T, Brandl A, Rasch S, et al. Fungal peritonitis: underestimated disease in critically ill patients with liver cirrhosis and spontaneous peritonitis. PLoS One. 2016;11(7):e0158389.

25. Merli M, Lucidi C, Di Gregorio V, et al. The spread of multi drug resistant infections is leading to an increase in the empirical antibiotic treatment failure in cirrhosis: a prospective survey. PLoS One. 2015;10(5): $\mathrm{e} 0127448$.

26. Ison MG. Empiric treatment of nosocomial spontaneous bacterial peritonitis: one size does not fit all. Hepatology. 2016;63:1083-1085.

27. Guarner C, Soriano G. Spontaneous bacterial peritonitis. Semin Liver Dis. 1997; 17:203-217.

28. Piroth L, Pechinot A, Di Martino V, et al. Evolving epidemiology and antimicrobial resistance in spontaneous bacterial peritonitis: a two-year observational study. BMC Infect Dis. 2014;14:287.

29. Choi SH, Soo Kim Y, Chung JW, et al. Clinical significance of untreated Candida species isolated from ascites in cirrhotic patients. Scand J Infect Dis. 2004;36:649-655.

30. Ríos-Toro JJ, Márquez-Coello M, García-Álvarez JM, et al. Soluble membrane receptors, interleukin 6 , procalcitonin and $\mathrm{C}$ reactive protein as prognostic markers in patients with severe sepsis and septic shock. PLoS One. 2017;12(4):e0175254.

31. Lee SS, Min HJ, Choi JY, et al. Usefulness of ascitic fluid lactoferrin levels in patients with liver cirrhosis. BMC Gastroenterol. 2016;16:132.

32. Dang Y, Lou J, Yan Y, et al. The role of the neutrophil Fcy receptor I (CD64) index in diagnosing spontaneous bacterial peritonitis in cirrhotic patients. Int J Infect Dis. 2016;49:154-160.

33. Yang Y, Li L, Qu C, et al. Diagnostic accuracy of serum procalcitonin for spontaneous bacterial peritonitis due to end-stage liver disease: a meta-analysis. Medicine. 2015;94:e2077.

34. Fernandes SR, Santos P, Fatela N, et al. Ascitic calprotectin is a novel and accurate marker for spontaneous bacterial peritonitis. J Clin Lab Anal. 2016;30:1139-1145.

35. Abdel-Razik A, Mousa N, Elhammady D, et al. Ascitic fluid calprotectin and serum procalcitonin as accurate diagnostic markers for spontaneous bacterial peritonitis. Gut Liver. 2016;10:624-631.

36. Maldonado J, González-Guerrero AB, Domínguez C, et al. Label-free bimodal waveguide immunosensor for rapid diagnosis of bacterial infections in cirrhotic patients. Biosens Bioelectron. 2016;85:310-316.

37. Vincent JL, Brealey D, Libert N, et al. Rapid diagnosis of infection in the critically ill, a multicenter study of molecular detection in bloodstream infections, pneumonia, and sterile site infections. Crit Care Med. 2015;43:2283-2291

38. van den Kieboom CH, Ferwerda G, de Baere I, et al. Assessment of a molecular diagnostic platform for integrated isolation and quantification of mRNA in whole blood. Eur J Clin Microbiol Infect Dis. 2015; 34:2209-2212.

39. Dellit TH, Owens RC, McGowan JE Jr, et al. Infectious Diseases Society of America; Society for Healthcare Epidemiology of America. Infectious Diseases Society of America and the Society for Healthcare Epidemiology of America guidelines for developing an institutional program to enhance antimicrobial stewardship. Clin Infect Dis. 2007;44(2):159-177. 


\section{Publish your work in this journal}

Therapeutics and Clinical Risk Management is an international, peerreviewed journal of clinical therapeutics and risk management, focusing on concise rapid reporting of clinical studies in all therapeutic areas, outcomes, safety, and programs for the effective, safe, and sustained use of medicines. This journal is indexed on PubMed Central, CAS,

EMBase, Scopus and the Elsevier Bibliographic databases. The manuscript management system is completely online and includes a very quick and fair peer-review system, which is all easy to use. Visit http://www.dovepress.com/testimonials.php to read real quotes from published authors.

Submit your manuscript here: http://www.dovepress.com/therapeutics-and-clinical-risk-management-journal 